\title{
Elective surgery in the context of the SARS COV- 2 pandemic: reintroducing delayed surgeries
}

\section{Editorial}

In December 2019 it was called to attention an outbreak of cases of atypical pneumonia in the Wuhan province in China. The first epidemiological reports showed that the infection had a highly contagious rate and it was lethally affecting a group of patients with risk factors; those who have previous chronic medical conditions. In January and February of 2020, the disease was propagated in several regions of China, and after that in the rest of continents starting in Europe, Asia and finally in the Americas. Finally on March 11, the World Health Organization (WHO) declared the pandemic of a new infectious disease called COVID-19. ${ }^{1,2}$ This new disease scenario, the health systems, especially the hospitals that have experienced more pressure because of the high demand of attention of patients with COVID, led to the modification of the structure of the hospitals and the process in such a way that the conventional program of scheduled surgeries was affected. In Mexico, several hospitals were selected for COVID patient attention, in those, elective surgery was prioritized for oncology, cardiovascular surgery, acute isquemia and surgical emergencies. ${ }^{3,4}$

The original purpose to cancel scheduled surgeries was to release space and health professionals to provide attention to the high demand of COVID patients, also to minimize the risk of infection of the patients, relatives and health care providers. However, recently it has been known that the mortality and morbidity rate is higher in surgical patients that had COVID during the perioperative period, which confirms that the right decision was to cancel the elective surgeries. Several authors had been shown enough evidence about this matter, like Doglietto, et al (2020) who analysed a retrospective cohort of 123 patients $41(33.3 \%)$ positive to COVID-19 and $82(66.7 \%)$ in the group control. Both groups without significant differences in age, gender, illness and comorbidities. They reported significantly higher mortality in the COVID-19 positive patient group (19.2\%) versus (2.4\%) the group control [OR: 9.5; 95\% IC, 1.77 - 96.53]. Pulmonary complications were significantly more frequent in the group of patients positive for COVID-19 versus the group control [OR: 35.63; $95 \%$ IC, 9.34 - 205.55]. ${ }^{1}$ Secondly, in a collaborative international study done by COVIDSurgcollaborative, they examined the data of 1,128 patients in 235 hospitals of 24 countries of Europe, Africa, Asia and Northamerica. The global mortality after 30days was $23.8 \%$. Elective surgery $18.9 \%$ and emergency surgery $25.6 \%$. In minor surgery, abdominal wall surgery was $16.3 \%$; in major surgery, orthopedic and cancer it was $26.9 \%$. The mortality rate was significantly higher in men $(28.4 \%)$ than women $(18.2 \%)$; in $>70$ years $(33.7 \%)$, versus $\prec$ 70 years $(13.9 \%)$. The most frequent complication was pneumonia $(51 \%)$ with acute respiratory distress syndrome that required mechanical ventilation. The death cause in $81.7 \%$ of the deaths were pulmonary complications. ${ }^{2}$ In the last year other authors contributed with more evidence in the same issue. The findings of these studies suggest that the immune system response due to surgical trauma as an aggregate factor in COVID-19 patients can increase the mortality and morbidity in these groups of patients. This can be explained because as the SARS CoV-2 and the surgical trauma induced proinflammatory response and deregulation of the immune system hence both
Volume 12 Issue 5 - 202 I

\author{
Juan Carlos Arana Reyes,' Rodolfo Aparicio \\ Ponce ${ }^{2}$ \\ 'Department of Surgery, Anahuac Mayab University, Mexico \\ ${ }^{2}$ Department of Surgery, Regional High Specialty Hospital of the \\ Yucatan Peninsula, Mexico
}

Correspondence: Dr. Juan C Arana, Department of Surgery, Anahuac Mayab University, Mexico, Tel 52 99935I 2096. Email juan.arana1974@gmail.com, juan.arana@anahuac.mx

Received: August 25, 202 I | Published: September 03, 2021

summation effects increases the mortality and morbidity postsurgical risk; ${ }^{3}$ additionally, the hospital environment exposes the patient to the risk of infection and the deleterious effects of mechanical assisted ventilation can deteriorate the pulmonary response. Before the SARS $\mathrm{CoV} 2$ pandemic, pulmonary complications rate were reported in $10 \%$ and the mortality in $3 \%$ in postoperative period. ${ }^{2}$

Over the course of the pandemic surgical emergencies have been prioritized, as well the cases in which the natural history of the disease justified the risk- benefits versus risk of admission to the hospital. However, there are still some cases in which the evolution of the disease may cause the surgery to be done in the months to come. The planning of reactivation of the surgical services for elective surgeries must be developed in such a way that the patients' and family safety must be guaranteed, the health care providers and the facilities, in addition must make the most of the available resources. So, we present some recommendations for this particular situation.

Demand analysis. The hospitals that are being affected in their programs of elective surgeries have a delayed number of patients waiting for the procedures. In the same way, the reference system between the first, second and third level of health care attention has a high number of cases accumulated because of the pandemic. The reintroduction elective surgery plan must consider the capacity of the surgical productivity to solve the delayed surgeries as a result of the pandemic, but at the same time, to solve the new cases without leaving out the delayed cases.

Demand of the facilities capacity. With the main goal to reduce the delayed surgeries because of the pandemic, in this section, the capacity of the surgical services productivity must be analyzed, so that a plan would be projected for the initial calculated capacity at about $100 \%$, until that of $50 \%$ for each operating room. For this one needs to consider the possibility to increase the capacity of the facility as well as the structure and the procedures. 
Standardization of mechanisms to prioritize cases. In Mexico, the Mexican Institute of Social Security, one of the better organized health institutions during the pandemic suggested 3 criteria: 1.Disease evaluation: non-surgical alternative, feasibility to delay the surgical procedure without deterioration of patient health; 2.- Surgical procedure evaluation: estimated surgical time, estimated hospital stay, possibility to require stay in the intensive care unit, bleeding risk, surgical site considering that following surgical procedures as head, neck, thorax and upper abdominal, are considered High Risk Infection for health care providers; and 3.- Patient condition evaluation: age, body mass index, comorbidities, COVID - 19 contacts in the last 14 days. Some cases are prioritized whose "additional delaying" puts the patient at risk; for those who require less surgical time, those who require less postsurgical hospital stay; and for those who have less risk to need intensive care unit services. With all this the programs of elective surgeries can be optimized.

Implementation of safety measures. In the HRI procedures the QPCR for SARS - COV test must be requested. The safety measures must include: a) risk factor questionnaire to select patients with infection risk [asymptomatic or pre - clinical]; b) RT - qPCR for SARS - COV2 test for the patient with high risk infection; c) Chest $\mathrm{X}$ - ray or chest CT scan 72 hours before the procedure, as necessary; and d) Use of personal protective equipment. Note: For the surgical emergencies that patients are suspects' cases, with questionnaire positive or high risk infection, the surgical team must use the personal protective equipment also for the cases that have been confirmed positive to COVID - 19.,

The planning of surgical services must be collegiate and multidisciplinary. It is very important to affirm that in situations of crisis this planning must be reviewed continuously, well situations like this new variants of COVID - 19, and the development of new vaccines or new medications could modify the original planning. And finally, it should not be ignored that the patient positive to COVID-19 regardless of the time of infection, would require interdisciplinary treatment with the participation of Pneumology, Internal Medicine, Intensive Care, Infectology, Surgical and Nurse Services.

\section{Acknowledgments}

None.

\section{Conflicts of interest}

The authors declare no conflicts of interest related to this article.

\section{Funding}

None.

\section{References}

1. Doglietto F, Vezzoli M, Gheza F, et al. Factors Associated with Surgical Mortality and Complications Among Patients with and without Coronavirus Disease 2019 (COVID-19) in Italy. JAMA Surg. 2020;55(8):691-702.

2. COVID Surg Collaborative. Mortality and pulmonary complications in patients undergoing surgery with perioperative SARS-COV2 infection: an international cohort study. The Lancet. 2020;396:27-38.

3. Besnier E TJSL. We asked the experts: Covid-19 Outbreak: is there still a place for scheduled surgery? Reflection from pathophysiological data. World J Surg. 2020;44(6):1695-1698.

4. Plan of the activities upgrade for the reintroducing of delayed health services for the COVID- 19 contingency. Department of medical services; Medical Attention Unit. Report No. N 09552461 2C00/000323. Mexico: Social Security Mexican Institute; 2020

5. Plan of the activities upgrade for the reintroducing of delayed health services for the COVID- 19 contingency. Department of medical services. Mexico: Social Security Mexican Institute; 2020. 